\title{
Correction to: COVID-19 and output in Japan
}

\author{
Daisuke Fujii ${ }^{1,2}$ (D) Taisuke Nakata ${ }^{1}$
}

Published online: 14 October 2021

(C) Japanese Economic Association 2021

\section{Correction to: The Japanese Economic Review https://doi.org/10.1007/s42973-021-00098-4}

In this article the affiliation 'RIETI (Research Institute of Economy, Trade and Industry)' for Dr. Fujii was missing.

Moreover, in this article the following acknowledgment was missing.

Daisuke Fujii is supported by JSPS Grant-in-Aid for Scientific Research (KAKENHI), Project Numbers 17K13744 and 20K13475, and Research Institute of Economy, Trade and Industry (RIETI). This paper was previously published as RIETI Discussion Paper Series 21-E-004.

The original article can be found online at https://doi.org/10.1007/s42973-021-00098-4.

Daisuke Fujii

dfujii@e.u-tokyo.ac.jp

Taisuke Nakata

taisuke.nakata@e.u-tokyo.ac.jp

1 Faculty of Economics, University of Tokyo, 7-3-1 Hongo, Bunkyo-ku, Tokyo 113-0033, Japan

2 RIETI (Research Institute of Economy, Trade and Industry), Tokyo, Japan 